\title{
The research of event-based intelligent prediction with minimum energy consumption of wireless sensor network node state transition strategy
}

\author{
Jingzhao Li and Dayu Yang*
}

College of Electrical and Information Engineering, Anhui University of Science and Technology, Huainan, 232001, China

Email: jzhli@aust.edu.cn

Email: 1097956908@qq.com

${ }^{*}$ Corresponding author

\section{Ping Ren and Zhijun Zhang}

College of Computer Science and Engineering, Anhui University of Science and Technology, Huainan, 232001, China

Email: 37880863@qq.com

Email:664195764@qq.com

\begin{abstract}
Whether the state transition of wireless sensor network (WSN) nodes that are based on energy-efficient can be carried out is related to the parameters of the subsequent event's occurrence such as time, and a previous estimation of the time for the uncertain events' occurrence is necessary. In this paper, we proposed a grey multistage model with a buffer operator is utilised to get the distance between nodes, and the delivered information is predicted by PSO algorithm and is amended by Kalman filtering, then, the fuzzy reasoning based on vague set is applied to get the time value of sensor networks' uncertain events. The state transition of Wireless Sensor Networks' nodes is controlled by the time values of uncertain events and the input signal. So that the Wireless Sensor Networks' nodes can get into the optimal working mode and achieve the purpose of extending the life cycle by saving the node's energy.
\end{abstract}

Keywords: energy-efficient scheduling; event time; node state transition; wireless sensor network; WSN.

Reference to this paper should be made as follows: Li, J., Yang, D., Ren, P. and Zhang, Z. (2017) 'The research of event-based intelligent prediction with minimum energy consumption of wireless sensor network node state transition strategy', Int. J. Ad Hoc and Ubiquitous Computing, Vol. 25, No. 4, pp.204-212.

Biographical notes: Jingzhao Li received his MA from China University of Mine and Technology in 1992 and PhD in the Key Lab of Power Electronics and Power Drives at Hefei University of Science and Technology, in 2003. He is currently a Professor with the College of Electrical and Information Engineering, Anhui University of Science and Technology, China. His research interests include computer control, internet of things technology and embedded systems.

Dayu Yang received the Bachelor's of Engineering in the School of Communication Engineering, Huaibei Normal University in 2013, China. Now, he is a PhD candidate of Electrical and Information Engineering, AUST. His current research interests are in natural language processing, data mining and wireless network.

Ping Ren received the Bachelor's of Engineering in the School of Anhui University Science and Technology, China. Now, she is a PhD candidate of Computer and Science, AUST. Her current research interests are in natural language processing, data mining and internet of things.

Zhijun Zhang received the Bachelor's of Electrical Engineering in the school of 2015, China. Now, he is a Master degree candidate of Electrical and Information Engineering, AUST. His current research interests are in natural language processing, data mining and internet of things. 


\section{Introduction}

Wireless sensor network (WSN) is made up of a set number of nodes with MCU and low power micro sensor in the way of self-organisation. WSN can monitor the targets and collect the information from the environment through the cooperation of a large amount of sensors, which will not only get all those information processed but also send users who are in need those detailed and accurate data acquired. In recent years, with the extensive use of WSN in different fields such as national defence and military, healthcare, environmental monitoring, etc., WSN has got further developments. At present, much research of WSN (Eu et al., 2011) have focused on the extension of the network lifetime. Since the volume of sensor nodes are generally small (Bein et al., 2011), mainly rely on battery power, and portable battery power is limited. Under normal conditions, WSN is applied in a harsh environment (Raghuram and Venkatesh, 2012) and it has a large number of nodes. After consuming the limited node energy, it cannot get supplements in time. In addition (Ciszkowski et al., 2011; Tan et al., 2012), periodic replacement of the battery is inconvenient. Therefore, in the premise of ensuring the communication efficiency of the entire WSN, saving node energy plays an important role in extending the life cycle of the entire WSN. Energy consumption in WSN is mainly from the wireless communication transmission (Molina and Alba, 2011), and energy consumption of transmitting one bit data in nodes is much larger than that during processing information. In connection with the feature, how to save and balance the energy in nodes has become one of main directions of WSN research.

To prolong the network life time and optimise the throughput between the wireless sensors with stringent power, the biggest difficulty is that the working state of sensors. Actually, the working state of sensor is related to the parameters of the next event's occurrence such as time, communication distance, and transmitting information or other parameters. To solve this problem, the sensor need alternate optimal working mode under a certain condition, we put forward the using of vague set fuzzy get the uncertain time value of events, multistage grey model to calculate the distance between nodes, PSO algorithm and Kalman filtering amend the amount of information. Finally realising the choice of node working mode.

The rest of the paper is organised as follows. In Section 2, we will first introduce and classify different models of wireless sensor node. Next, in Section 3, we propose the corresponding four modules of WSN nodes, which were working under the nine working state. In Section 4, in order to predict the occurrence of unknown event, we formulate the PSO mechanism model and amended by Kalman filtering. In Section 5, we combine with buffer operator, multistage grey model to estimate the distance between nodes and the judgement standard is given. In Section 6, we get the occurrence time of unknown event by using vague set fuzzy inference. On the basis of event trigger signal and data transmission between nodes event time signal, we put forward the nodes' state control mechanism, and by a state diagram representation in Section 7. Finally, we conclude the paper in Section 8.

\section{Analysis of the energy consumption of wireless sensor node}

In WSN, there are many reasons for consuming energy, such as conflicts, overhearing, expenditure of controlling message, idle listening, fluctuation of the load, inappropriate transmission, data redundancy and so on. However, primary energy consumption is still that the nodes working in inappropriate condition. Under what conditions that the nodes get into hibernation while saving more energy than maintaining a normal state is an important problem.

In recent years, there are so many researches for the energy consumption of wireless sensor nodes at home and abroad. For instance, Kamyabpour and Hoang (2012) proposed that the energy consumption of nodes can be predicted by the Markov chain and established the model of node energy consumption based on state transition. What's more, the model will not influence by factors like the topological structure, it is a universal node energy consumption model. Rizvandi et al. (2011) calculated the energy consumption of the whole network by tracking the entire process of data stream. Then they proposed an energy consumption model based on tracking data stream. It can calculate the energy consumption starting from the data stream. The model will not influence by dynamic node status and network communication sequence. In addition, it optimised topology, power and routing designs in chain and cluster. So, an optimisation model of the network based on energy consumption was established. It will shorten the life of WSN that without a good management of spare parts. To extend the life cycle of WSN, Bakr and Lilien (2011) modified the protocol, which provides a best and energysaving management of spare parts in WSN node, including reserve choice and so on. In addition, a quantitative comparison of life and energy consumption between the two protocols has been done. A greedy heuristic project for solving attack was proposed by Sun and Shayman et al. (Sun and Shayman, 2010), which solved a problem of implementing the transmission of nodes' adaptive data by making the most of the life cycle of WSN by using of a method that combined relay deployment and the mixed integer non-linear programming method of adaptive data transmission. The numerical simulation results are shown that a $10 \%$ increase in additional relay nodes can extend network lifetime by 50\%. Ehsan and Hamdaoui (2012) used the weighted average algorithm based on the smallest hop technology, and built an energy consumption model of the square network. They derived the expressions of energy consumption while regarding the energy consumption of sending and receiving data as the primary. And it solved the fuzzy problem of energy consumption expression. Girban and Popa (2010) deduced the energy consumption models of the common nodes, the gathering nodes and the cluster head 
nodes based on mode of one hop transmission in sensor networks. Kamyabpour and Hoang (2010) divided node into two states: work and sleep. They also ideally built the model of node energy consumption and the model of network energy consumption. In addition, they gave the probability of the nodes to send data in the model and other specific parameters. These studies above are mainly based on the protocol of sensor node transportation, network layout, node management, dynamic energy management technology (Bhatti et al., 2010) and dynamic voltage adjustment technology for energy consumption of different modules. In this paper, we will work from the energy consumption of the overall state while each module working together in node. Through controlling the state transition of the wireless sensor node, it can get the WSN node into optimal working mode, at the same time it can extend the using life by saving energy.

\section{Analysis of the module work mode and transition time of node's working state}

\subsection{Analysis of WSN module work mode}

The radio frequency communication module of wireless sensor nodes has the following six working mode:

1 receiving mode: its relative power consumption is about $10^{6}$

2 sending mode: its relative power consumption is about $10^{6}$

3 listening mode: its relative power consumption is about $10^{6}$

4 idle mode: its relative power consumption is about $5 \times 10^{4}$

5 sleep mode: its relative power consumption is about $10^{3}$

6 off mode: its relative power consumption is about 1.

The power consumption did not appear to be much different from the first three modes, the power consumption of sending mode is slightly larger than receiving mode in some radio frequency modules, but some are on the contrary. In some cases, the power consumption of listening mode is slightly smaller than that of sending or receiving mode. But they all have little difference, so these three boils can be put down to one order of magnitude here.

The microprocessor module of wireless sensor nodes has the following three working mode:

1 running mode: its relative power consumption is about $5 \times 10^{4}$

2 idle mode: its relative power consumption is about 30

3 sleep mode: its relative power consumption is about 1 .

The sensor module of wireless sensor nodes has the following two working modes:
1 operating mode: its relative power consumption is about $2 \times 10^{3}$

2 off mode: its relative power consumption is about 1 .

The memory module of wireless sensor nodes has the following three working modes:

1 active mode: its relative power consumption is about $10^{4}$

2 idle mode: its relative power consumption is about 20

3 sleep mode: its relative power consumption is about 1 .

The above four modules work together, and there are nine kinds of overall working state of WSN nodes that are composed of them. The corresponding relationship between nine kinds of working state and the working mode of four corresponding modules is showed in Table 1.

Table 1 The overall working state of WSN nodes

\begin{tabular}{lll}
\hline $\begin{array}{l}\text { The overall } \\
\text { working state of } \\
\text { nodes }\end{array}$ & $\begin{array}{l}\text { The working mode } \\
\text { of radio frequency } \\
\text { communication module }\end{array}$ & $\begin{array}{l}\text { The working mode } \\
\text { of microprocessor } \\
\text { module }\end{array}$ \\
\hline$S_{0}$ & Send-receive & Running \\
$S_{1}$ & Receive & Running \\
$S_{2}$ & Idle & Running \\
$S_{3}$ & Receive & Idle \\
$S_{4}$ & Listening & Idle \\
$S_{5}$ & Sleep & Working \\
$S_{6}$ & Sleep & Sleep \\
$S_{7}$ & Sleep & Sleep \\
$S_{8}$ & Off & Sleep \\
& & \\
\hline The working & The working mode & The working mode \\
mode of memory & of radio frequency & of microprocessor \\
module & communication module & module \\
\hline$S_{0}$ & Send-receive & Running \\
$S_{1}$ & Receive & Running \\
$S_{2}$ & Idle & Running \\
$S_{3}$ & Receive & Idle \\
$S_{4}$ & Listening & Idle \\
$S_{5}$ & Sleep & Working \\
$S_{6}$ & Sleep & Sleep \\
$S_{7}$ & Sleep & Sleep \\
$S_{8}$ & Off & Sleep \\
\hline & &
\end{tabular}

\subsection{Analysis of transition time of node's working state}

State transition takes the time and energy. The deeper the nodes in a dormant state, the more time that the nodes turn to a fully working state or a shallow sleep state will need, and the more energy will consume.

Assume that the node used to work in the working state of relatively high-energy consumption, the energy that 
needs to consume is $P_{h}$, and it needs to turn to a low-energy state which the energy consumption is $P_{l}$ from high-energy state at time $t_{0}$.

If the wireless sensor nodes are in the working state and there are events occurring at time $t_{1}\left(t_{1}>t_{0}\right)$, the total energy consumption of the nodes that working in high-energy state will be:

$$
E_{h}=P_{h}\left(t_{1}-t_{0}\right)
$$

If the switching time that the sensor nodes transfer from high-energy states to low-energy states is $\tau_{c 1}$, the total energy that consumed during the time will be:

$$
\begin{aligned}
E_{l} & =0.5 \tau_{c 1}\left(P_{h}+P_{l}\right)+\left(t_{1}-t_{0}-\tau_{c 1}\right) P_{l} \\
& =0.5 \tau_{c 1} P_{h}+\left(t_{1}-t_{0}-0.5 \tau_{c 1}\right) P_{l}
\end{aligned}
$$

The saved energy in this transition is:

$$
E_{s}=E_{h}-E_{l}=\left(t_{1}-t_{0}-0.5 \tau_{c 1}\right)\left(P_{h}-P_{l}\right)
$$

Now the problem is: when events need to be addressed, wireless sensor nodes must transfer from low-energy states to high-energy states.

Let the necessary time of state transition from the low energy to high energy is $\tau_{c 2}$, and the average energy assumption is $0.5\left(P_{h}+P_{l}\right)$, so the extra energy in this stage will be:

$$
E_{e}=0.5 \tau_{c 2}\left(P_{h}+P_{l}\right)
$$

Obviously, in order to save energy consumption while transferring from high-energy states to low-energy states, there are some conditions must be needed:

$$
E_{s}-E_{e}>0
$$

that is:

$$
\left(t_{1}-t_{0}-0.5 \tau_{c 1}\right)\left(P_{h}-P_{l}\right)-0.5 \tau_{c 2}\left(P_{h}+P_{l}\right)>0
$$

Thus:

$$
T_{\min }=t_{1}-t_{0}>0.5 \tau_{c 1}+0.5 \tau_{c 2}\left(P_{h}+P_{l}\right) /\left(P_{h}-P_{l}\right)
$$

When WSN nodes doing state transition, it is the minimum interval before the next event happens. Obviously, the larger that the interval is, the more energy will be stored.

According to the discussion above, whether the state transition of WSN's nodes that are based on energy efficient can be carried out is related to the time parameters of the subsequent event's occurrence (or other parameters). Some of these events are under a certain time of occurrence. For instance, the node completes the task of acquiring data by executing its own sensor. And some of these events do not have a certain time of occurrence. For example, when the entire WSN nodes are regarded as other relaying nodes to transmit the flow from the others, the time of occurrence will be uncertain. So, in order to save the energy of the network nodes and let it get into the optimal working mode, a previous estimation of the time that the uncertain events' occurrence is necessary. Through the simulation and experimental analysis, the time of the uncertain events' occurrence is mostly related with the distance of the node and its' surroundings that can be perceived. Moreover, it is influenced by the amount of transmitting information or other parameters. Here, the amount of information to be delivered is predicted by PSO and is amended by Kalman filtering. A grey multistage model with a buffer operator is utilised to get the distance between nodes. Approximate reasoning based on vague set is applied to reasoning the time values of sensor networks' uncertain events. The state transition of WSNs' nodes is controlled by the time values of uncertain events and certain events as well as the input signal.

\section{Predicting event information based on PSO and amending event information by Kalman filtering}

\author{
(3)
}

PSO algorithm is a kind ô initelligent computing technology. It has a lot of advantages such as a simple algorithm concept and easy to realise. So, it got a great success during a short time in various aspects like pattern recognition, function optimisation, etc. In this paper, the author will optimise the event information of WSN by PSO.

There are $N$ nodes in the event information group, and they are composed of $M$ particles. These particles spread between $N$ nodes, so, the position information of the $i$ th information particle at time in the event information group is:

$$
w_{i}^{k}=\left(w_{i 1}^{k}, w_{i 2}^{k}, \ldots, w_{i N}^{k}\right)^{T}
$$

Among them: $w_{i N}^{k} \in\left[w_{U N}, w_{D N}\right], w_{U N}, w_{D N}$ is, respectively, represented upper and lower limits in the event information group.

The speed of the $i$ th information particle at time $k$ in the event information group is:

$$
v_{i}^{k}=\left(v_{i 1}^{k}, v_{i 2}^{k}, \ldots, v_{i N}^{k}\right)^{T}
$$

where $v_{i N}^{k} \in\left[v_{\min }, v_{\max }\right], \quad v_{\min }, \quad v_{\max }$ is, respectively, represents the minimum and maximum speed in the event information group.

The optimal position of single-nodes in the event information group is:

$$
w_{i}^{k}=\left(w_{i 1}^{k}, w_{i 2}^{k}, \ldots, w_{i n}^{k}\right)^{T}
$$

The optimal position of all the nodes in the event information group is:

$$
w_{g}^{k}=\left(w_{g 1}^{k}, w_{g 2}^{k}, \ldots, w_{g n}^{k}\right)^{T}
$$

Among them: $1 \leq n \leq N, 1 \leq i \leq M$.

Then the event information variable of speed and position will be updated at the next time by the following formula:

$$
\begin{aligned}
& v_{i d}^{k+1}=\omega v_{i d}^{k}+c_{1} r_{1}\left(w_{i d}^{k}-x_{i d}^{k}\right)+c_{2} r_{2}\left(w_{g d}^{k}-x_{i d}^{k}\right) \\
& w_{i d}^{k+1}=w_{i d}^{k}+v_{i d}^{k+1}
\end{aligned}
$$


Then the state equation of event information system will be obtained:

$$
Q=A(k) W(k)+B(k) v(k)
$$

Then the prediction equation will be obtained by amending it with Kalman filtering:

$$
\left\{\begin{array}{l}
Q^{\prime}(k \mid k-1)=A(k-1) Q^{\prime}(k-1 \mid k-1) \\
P(k \mid k-1)=A(k-1) P(k-1 \mid k-1) A^{T}(k-1)+J
\end{array}\right.
$$

To avoid errors of algorithm caused by data redundancy, a 'forgetting' factor is introduced in prediction, so that the past data can be forgot. The prediction equation (14) is amended as:

$$
\left\{\begin{array}{l}
Q^{\prime}(k \mid k-1)=A(k-1) Q^{\prime}(k-1 \mid k-1) \\
P(k \mid k-1)=\lambda(k) \cdot A(k-1) P(k-1 \mid k-1) A^{T}(k-1)+J
\end{array}\right.
$$

The forgetting factor $\lambda(k) \geq 1$ in formula (15).

The initial conditions of the system status, which are based on the estimation of unknown nodes in the event information given above, and doing iterative calculation in the process of predicting and correction, setting the accuracy $\varepsilon$ in advance, when meet the condition:

$$
\sqrt{Q^{\prime 2}(k)+Q^{\prime 2}(k \mid k-1)} \leq \varepsilon
$$

When the iteration end. This will give a more accurate event information $Q^{\prime}$.

\section{Estimate the nodes distance by multistage grey model with a buffer operator}

Theoretical analysis and experimental verification all show that: WSN transmission always influenced by many factors (Incel et al., 2011), such as surrounding dielectric coefficient, environmental reflection coefficient, conduction effect of wireless electromagnetic wave, etc. Therefore, the distance formula that based on the strength of send and receive signals in wireless network nodes is showed in formula (17).

$$
l=A \times 10^{\frac{(C-q)}{10 n}}
$$

In formula (17), the radio frequency parameter $C$ that is represented by $\mathrm{dBm}$ is defined as the absolute value of the average energy received when $1 \mathrm{~m}$ away from the transmitter, the propagation factor or propagation coefficient $n$ related to wireless network environment, which reflects the rate of decay of the signal energy while increasing the distance to the transceiver. $q$ represents signal strength of the received power in nodes, it is measured by experiment in the actual environment.

The distance between nodes can be got from formula (17). In many cases, the distance value needs to obtain a certain precision. Following the distance between nodes as the input, establish a RSSI distance algorithm, which based on multistage grey model.
According to analysing small amount of partially known data, the grey system can find valuable information, so it can finding out the inherent regularity of the system, these regular data must be able to provide the basis for the prediction of future short-term trends of systems. The generation of grey sequence is a data generating technique that can let the original data reflect the variation of the system. If the system itself is influenced by the external environment and the data are erratic. Prediction of grey model will become worse. Therefore, in order to solve the problem of the disturbance of system information prediction, a buffer operator is introduced.

$L D$ represents the buffer operators have worked on the original data one time, $L D_{m}(m=1,2, \ldots, n)$ represents many times. The size of $m$ the value of is set based on the actual situation of the distribution of wireless sensor nodes. Finally, prediction data of node distance will be reduced. The calculation is as follows:

Assume that the sequence of original data is:

$$
L^{0}=\left(l_{1}^{0}, l_{2}^{0}, \ldots, l_{n}^{0}\right)
$$

The buffer operator is $D$, after $l_{n}^{0^{\prime}}=l_{n}^{0} D$, the new sequence $L^{0^{\prime}}$ will be got:

$$
L^{0^{\prime}}=\left(l_{1}^{0^{\prime}}, l_{2}^{0^{\prime}}, \ldots, l_{n}^{0^{\prime}}\right)
$$

Let

$$
L D^{2}=L D D=\left(l(1) d^{2}, l(2) d^{2}, \ldots, l(n) d^{2}\right)
$$

Among them:

$$
\begin{array}{r}
l(k) d^{2}=\frac{1}{n-k+1}[l(k) d+l(k+1) d+\cdots+l(n) d] \\
k=1,2, \ldots, n
\end{array}
$$

$L^{1^{\prime}}$ is generated by the first-order buffer operator:

$$
L^{1^{\prime}}=\left(l_{1}^{1^{\prime}}, l_{2}^{1^{\prime}}, \ldots, l_{n}^{1^{\prime}}\right)
$$

Among them: $l_{k}^{1^{\prime}}=\sum_{i=1}^{k} l_{i}^{0^{\prime}}, \quad k=1,2, \ldots, n$

Test the smoothness of the generation sequence $L^{1^{\prime}}$ :

$$
\rho_{k}=\frac{l_{k}^{1^{\prime}}}{\sum_{i=1}^{k-1} l_{i}^{0^{\prime}}}
$$

The development coefficient $\alpha$ trusts in the smooth ratio $\rho_{k}$ of buffer sequence, it is showed in formula (24).

$$
\alpha=\frac{b / l_{k-1}^{1}-\rho_{k}}{1+\rho_{k} / 2} b
$$

The prediction model is:

$$
l_{i+1}^{0^{\prime \prime}}=l_{i+1}^{1^{\prime \prime}}-l_{i}^{1^{\prime \prime}}=\left(l_{1}^{\prime(0)}-\frac{b}{a}\right) \mathrm{e}^{-a k}, \quad k=1,2, \ldots, n
$$

Which the values of $a$ and $b$ can be obtained by the differential to $L^{1^{\prime}}$. 
When $k \geq n, l_{i+1}^{\prime \prime(k)}$ is the estimation of distance between nodes.

$$
T_{i}=R_{\max }\left(l^{\prime}, Q^{\prime}\right) \times\left(T_{\max }\right)_{i}
$$

\section{Obtain the time of uncertain event based on Vague sets fuzzy reasoning}

The prediction of the time of uncertain events in sensor networks using two-dimensional vague sets, the two input, respectively, are average distance $l^{\prime}$ between the node to be analysed and the nodes around it and the event information $Q^{\prime}$. The estimation time $T$, which presents next events occurred in fuzzy predictor output.

If the average distance between nodes and its surroundings is called $l$-the universe, $l^{\prime}=\left\{l_{1}^{\prime}, l_{2}^{\prime}, \ldots, l_{n}^{\prime}\right\}$, and $A$ represents vague set of the node distance, then $A$ can be respected by true membership function $t_{A}$ and false membership function $f_{A}$ :

$$
A=\sum_{i=1}^{n} \frac{\left[t_{A}\left(l_{i}^{\prime}\right), 1-f_{A}\left(l_{i}^{\prime}\right)\right]}{l_{i}^{\prime}}, \quad i=1,2, \ldots, n
$$

Among it:

$$
\begin{aligned}
& t_{A}\left(l_{i}\right)=\frac{1}{1+\left(l_{i}^{\prime}-a\right)^{2} / a}, \quad f_{A}\left(l_{i}\right)=\frac{\left(l_{i}^{\prime}-a\right)^{2} /(a+1)}{1+\left(l_{i}^{\prime}-a\right)^{2} /(a+1)}, \\
& a=\max \left\{l_{i}\right\}(i=1,2, \ldots, n)
\end{aligned}
$$

Similarly, if $Q^{\prime}$ is domain of discourse, besides $Q^{\prime}=\left\{Q_{1}^{\prime}, Q_{2}^{\prime}, \ldots, Q_{m}^{\prime}\right\}$, and $B$ represents vague set $\{$ The maximum deviation rate $\}$, then:

$$
B=\sum_{i=1}^{m} \frac{\left[t_{B}\left(Q_{i}^{\prime}\right), 1-f_{B}\left(Q_{i}^{\prime}\right)\right]}{Q_{i}^{\prime}}, \quad i=1,2, \ldots, m
$$

Among them:

$$
\begin{aligned}
& t_{B}\left(Q_{i}^{\prime}\right)=\frac{1}{1+\left(Q_{i}^{\prime}-b\right)^{2} / b}, \quad f_{B}\left(Q_{i}^{\prime}\right)=\frac{\left(Q_{i}^{\prime}-b\right)^{2} /(b+1)}{1+\left(Q_{i}^{\prime}-b\right)^{2} /(b+1)}, \\
& b=\max \left\{Q_{i}^{\prime}\right\}(i=1,2, \ldots, m)
\end{aligned}
$$

$l^{\prime}$ and $Q^{\prime}$ as two-input, $T$ is the inference rule of output: if $l^{\prime}$ and $Q^{\prime}$ then $T$.

The match facts of known rules $l^{\prime}$ and $Q^{\prime}$ both can be respected as Vague Set, $l_{i}^{\prime}$ means previous part of the rule, $Q_{i}^{\prime}$ means the match facts, among it, $l^{\prime}$ and $Q^{\prime}$, respectively, is:

$$
\begin{aligned}
& l_{i}^{\prime}=\left[\begin{array}{l}
l_{i 1}^{\prime} \\
l_{i 2}^{\prime}
\end{array}\right]=\left[\begin{array}{l}
{\left[t_{A 1}^{i}, f_{A 1}^{i}\right]} \\
{\left[t_{A 2}^{i}, f_{A 2}^{i}\right]}
\end{array}\right] \quad(i=1,2, \ldots, n) \\
& Q_{i}^{i}=\left[\begin{array}{l}
Q_{i 1}^{\prime} \\
Q_{i 2}^{\prime}
\end{array}\right]=\left[\begin{array}{c}
{\left[t_{B 1}^{i}, f_{B 1}^{i}\right]} \\
{\left[t_{B 2}^{i}, f_{B 2}^{i}\right]}
\end{array}\right] \quad(i=1,2, \ldots, m)
\end{aligned}
$$

The matching degree $R\left(l^{\prime}, Q^{\prime}\right)$ of $l^{\prime}$ and $Q^{\prime}$ is:

$$
\begin{aligned}
& R\left(l^{\prime}, Q_{i}^{\prime}\right)=\frac{1}{2} \sum_{j=1}^{2} M\left(V_{l^{\prime}}\left(u_{j}\right), V_{Q^{\prime}}\left(u_{j}\right)\right) \\
& =\frac{1}{2} \sum_{j=1}^{2} \begin{array}{r}
{\left[1-\left|s\left(V_{l^{\prime}}\left(u_{j}\right)\right)-s\left(V_{Q_{i}^{\prime}}\left(u_{j}\right)\right)\right| / 4\right.} \\
\left.-\left(t_{l^{\prime}}\left(u_{j}\right)-t_{Q_{i}^{\prime}}\left(u_{j}\right)\right)+\left|f_{l^{\prime}}\left(u_{j}\right)-f_{Q_{l}^{\prime}}\left(u_{j}\right)\right|\right] / 4
\end{array}
\end{aligned}
$$

In the formula (31): $M\left(V_{l^{\prime}}\left(u_{j}\right), V_{Q_{i}^{\prime}}\left(u_{j}\right)\right)$ is the degrees of similarity between $V_{l^{\prime}}\left(u_{j}\right)$ and $V_{Q_{i}^{\prime}}\left(u_{j}\right)$, among them, $V_{l^{\prime}}\left(u_{j}\right)$ and $V_{Q_{i}^{\prime}}\left(u_{j}\right)$ are Vague number.

$$
\begin{aligned}
& s\left(V_{l^{\prime}}\left(u_{j}\right)\right)=t_{j}-f_{j} ; \quad s\left(V_{Q_{j}^{\prime}}\left(u_{j}\right)\right)=t_{j}^{i}-f_{j}^{i} ; \\
& (i=1,2, \ldots, n ; j=1,2, \ldots, m)
\end{aligned}
$$

The larger $R\left(l^{\prime}, Q^{\prime}\right)$ is the higher the matching degree of $l^{\prime}$ and $Q^{\prime}$ will be. In the $n$ control rules, select the highest degree of matching $R_{\max }, \max \in(1,2, \ldots, n)$ which is the highest degree of matching and use the maximum time of occurrence to trigger the rule, the prediction of the occurrence of uncertain events in sensor networks is:

$$
T_{i}=R_{\max }\left(l^{\prime}, Q^{\prime}\right) \times\left(T_{\max }\right)_{i}
$$

The control method does not need to do processes repeatedly blurring, fuzzy reasoning, ambiguity resolution, etc. For each set of $l^{\prime}$ and $Q^{\prime}$, after blurring, similar reasoning measure by Vague Set is necessary, then you can get the clear value of the occurrence of uncertain events, Algorithm 1 presents the pseudo-code for the time of uncertain events.

Algorithm 1 The pseudo-code for the time of uncertain events

Input: the node's average distance $l^{\prime}$, the event information $Q^{\prime}$

Output: the estimation time $T$

1 for ( $l^{\prime}$ is the average distance between nodes) do \{

2 A represents Vague set of the node distance

3 set true membership function $t_{A}$ and false membership function $f_{A}$;

$4 \quad$ set $A=\left(t_{A}\left(l_{i}^{\prime}\right), f_{A}\left(l_{i}^{\prime}\right)\right)$ as the model;

$10 \quad 3$

12 for $\left(Q^{\prime}\right.$ is domain of discourse $)$ do \{

$13 B$ represents Vague Set(the maximum deviation)

14 set $B=\left(t_{B}\left(Q_{i}^{\prime}\right), f_{A}\left(Q_{i}^{\prime}\right)\right)$ as the model;

$15 \quad 3$

16 for ( $l_{i}^{\prime}$ means previous part of the rule) do

17 if (thematching degree $R\left(l^{\prime}, Q^{\prime}\right)$ is the highest ) then ?

18 the prediction time of the occurrence of uncertain events in sensor networks:

$19 ; \quad T_{i}=R_{\max }\left(l^{\prime}, Q^{\prime}\right) \times\left(T_{\max }\right)_{i}$

$20 \quad\}$

$21 \quad$ return $T$; 


\section{The input signal of nodes and the state transitions}

The working state transitions of wireless sensor nodes is mainly controlled by CPU, it includes that opening and closing the crystal oscillators, opening the radio frequency module, which is used to send and receive, closing radio frequency communication, opening and closing the radio frequency listening signal, opening and closing the data acquisition of sensors, entering dormant state, starting the timer, external interrupt response of CPU and overflow of timer, etc. The work of wireless sensor nodes is also related to RF signal, including the RF data completely sent command, sending less data signals in buffer by RF, receiving waiting command by $\mathrm{RF}$, receiving the signal of waiting for the buffer overflow by RF, receiving the end signal by RF data, etc. Moreover, the nodes' work is related to node data transmission and the sensor signals, including incident response of node data sending packet, event trigger of sensor excursion, incident response of node data receiving packet, etc. To facilitate the state transition, now each input signal of wireless sensor nodes are assigned in Table 2 .

Table 2

The input_signal distribution for wireless sensor nodes

\begin{tabular}{ll}
\hline Input_signal & Working state \\
\hline$I_{0}$ & CPU controlled crystal oscillator off \\
$I_{1}$ & CPU controlled crystal oscillator on \\
$I_{2}$ & CPU controlled RF communication off \\
$I_{3}$ & CPU controlled RF sending on \\
$I_{4}$ & CPU controlled RF receiving on \\
$I_{5}$ & CPU controlled RF listening on \\
$I_{6}$ & CPU controlled RF listening off \\
$I_{7}$ & CPU controlled data acquisition of sensors on \\
$I_{8}$ & CPU controlled data acquisition of sensors off \\
$I_{9}$ & CPU sleep command \\
$I_{10}$ & Sending complete command by RF data \\
$I_{11}$ & Sending less data signals in buffer by RF \\
$I_{12}$ & Receiving waiting command by RF \\
$I_{13}$ & Receiving the signal of waiting for the buffer \\
$I_{14}$ & overflow by RF \\
$I_{15}$ & CPU timer on \\
$I_{16}$ & Response of CPU external interrupt \\
$I_{17}$ & Incident response of node data sending packet \\
$I_{18}$ & Event trigger of sensor excursion \\
$I_{19}$ & Incident response of node data receiving packet \\
$I_{20}$ & Receiving the end signal by RF data \\
\hline & Overflow of CPU timer \\
\hline
\end{tabular}

\subsection{Analysis of WSN module work mode}

The working mode of each wireless sensor module contains that: receiving mode of RF communication module, sending mode, listening mode, idle mode, sleep mode and off mode; running mode of microprocessor module, idle mode and sleep mode, operating mode of sensor module, off mode; active mode of memory module in wireless sensor node, idle mode and sleep mode, etc. These modules work interrelate and form the overall working state of a WSN nodes State_signals $=\left\{S_{0}, S_{1}, \ldots, S_{8}\right\}$.

All kinds of working state transformation mainly depends on the event trigger signal from collaboration between each module in the node Input_signals $=\left\{I_{0}, I_{1}, \ldots, I_{20}\right\}$, and by the analysis data transmission between the sensor nodes and node event time signal mentioned above Time_signals $=\left\{T_{0}, T_{1}, \ldots, T_{\varepsilon}\right\}$.

According to the analysis of nodes, the relationship of node state transition can be showed in Table 3 .

Table 3 The node state transition $a$

\begin{tabular}{ll}
\hline Node state transition & Relative condition \\
\hline$S_{2} \rightarrow S_{0}$ & $C_{0}=I_{3} \cup I_{4} \cup I_{14} \cup I_{18}$ \\
$S_{0} \rightarrow S_{2}$ & $C_{1}=\left(I_{3} \cup I_{4} \cup I_{14} \cup I_{18}\right) \cap T_{a}$ \\
$S_{0} \rightarrow S_{1}$ & $C_{2}=I_{4} \cup I_{12} \cup I_{13} \cup I_{18}$ \\
$S_{1} \rightarrow S_{0}$ & $C_{3}=I_{3} \cup I_{16}$ \\
$S_{2} \rightarrow S_{1}$ & $C_{4}=\left(I_{4} \cup I_{12} \cup I_{13} \cup I_{18}\right) \cap($ not $) I_{20}$ \\
$S_{1} \rightarrow S_{2}$ & $C_{5}=\left(I_{2} \cup I_{19}\right) \cap T_{a}$ \\
$S_{1} \rightarrow S_{3}$ & $C_{6}=I_{20}$ \\
$S_{2} \rightarrow S_{3}$ & $C_{7}=\left(I_{4} \cup I_{12} \cup I_{13} \cup I_{18}\right) \cap I_{20}$ \\
$S_{3} \rightarrow S_{2}$ & $C_{8}=I_{2} \cap I_{19}$ \\
$S_{2} \rightarrow S_{4}$ & $C_{9}=I_{5}$ \\
$S_{4} \rightarrow S_{2}$ & $C_{10}=I_{6}$ \\
$S_{2} \rightarrow S_{5}$ & $C_{11}=I_{2} \cap I_{14} \cap T_{b}$ \\
$S_{5} \rightarrow S_{2}$ & $C_{12}=I_{15}$ \\
$S_{2} \rightarrow S_{6}$ & $C_{13}=I_{2} \cap I_{6} \cap I_{9} \cap T_{c}$ \\
$S_{6} \rightarrow S_{0}$ & $C_{16}=I_{3} \cup I_{4} \cup I_{16}$ \\
$S_{2} \rightarrow S_{7}$ & $C_{18}=I_{2} \cap I_{6} \cap I_{9} \cap T_{d}$ \\
$S_{7} \rightarrow S_{0}$ & $C_{21}=I_{3} \cup I_{4} \cup I_{16}$ \\
$S_{2} \rightarrow S_{8}$ & $C_{23}=I_{2} \cap I_{6} \cap I_{9} \cap T_{e}$ \\
$S_{8} \rightarrow S_{0}$ & $C_{26}=I_{3} \cup I_{4} \cup I_{16}$ \\
\hline &
\end{tabular}

The transition relationship of node is showed in Figure 1.

The rest relationship of node state transition can be showed in Table 4.

The transition relationship of node is showed in Figure 2. It is easy to realise by wireless sensor nodes' microprocessor, Algorithm 2 presents the pseudo-code for the transition relationship of node state. 
Table 4 The node state transition $b$

\begin{tabular}{ll}
\hline Node state transition & Relative condition \\
\hline$S_{0} \rightarrow S_{6}$ & $C_{14}=\left(I_{2} \cup I_{10} \cup I_{11}\right) \cap I_{7} \cap I_{17} \cap T_{c}$ \\
$S_{1} \rightarrow S_{6}$ & $C_{15}=\left(I_{3} \cap I_{16}\right) \cap I_{7} \cap I_{17} \cap T_{c}$ \\
$S_{6} \rightarrow S_{1}$ & $C_{17}=I_{4} \cup I_{12} \cup I_{13} \cup I_{18}$ \\
$S_{0} \rightarrow S_{7}$ & $C_{19}=\left(I_{2} \cup I_{10} \cup I_{11}\right) \cap T_{d}$ \\
$S_{1} \rightarrow S_{7}$ & $C_{20}=\left(I_{3} \cup I_{16}\right) \cap T_{d}$ \\
$S_{7} \rightarrow S_{1}$ & $C_{22}=I_{4} \cup I_{12} \cup I_{13} \cup I_{18}$ \\
$S_{0} \rightarrow S_{8}$ & $C_{24}=\left(I_{2} \cup I_{10} \cup I_{11}\right) \cap T_{e}$ \\
$S_{1} \rightarrow S_{8}$ & $C_{25}=\left(I_{3} \cup I_{16}\right) \cap T_{e}$ \\
$S_{8} \rightarrow S_{1}$ & $C_{27}=I_{4} \cup I_{12} \cup I_{13} \cup I_{18}$ \\
\hline
\end{tabular}

Algorithm 2 The pseudo-code for the transition relationship of node state

Input: the minimum time interval of the transition of nodes $T_{\min }$, the node's average distance $l^{\prime}$, the event information $Q^{\prime}$, the matching degree $R\left(l^{\prime}, Q^{\prime}\right)$, the event trigger signal from collaboration between each module in the node Input_signal $=\left\{I_{a}, I_{b}, \ldots, I_{20}\right\}$

Output: the overall working state of a wireless sensor network nodes

$1 \quad$ if $\left(R\left(l^{\prime}, Q^{\prime}\right)=R_{\max }\right)$ then \{

2 the prediction time of the occurrence of uncertain events in sensor networks:

$3 \quad T_{t}=R_{\text {IIIax }}\left(l^{\prime}, Q^{\prime}\right) \times\left(T_{\text {IIIax }}\right)_{t} ;$

43

10 else select the highest degree of matching $R_{\max }$, $\max \in(1,2, \ldots, n)$

12 do $\left\{T_{i}=R_{\max }\left(l^{\prime}, Q^{\prime}\right) \times\left(T_{\max }\right)_{i}\right.$;

$13 \quad 3$

14 for (each $T_{i}$ in set) do \{

15 State_signals $=($ Input_signals, Time_signals $)$

$16 \quad 3$

17 return State signals,

Figure 1 The diagram a of system running convert state

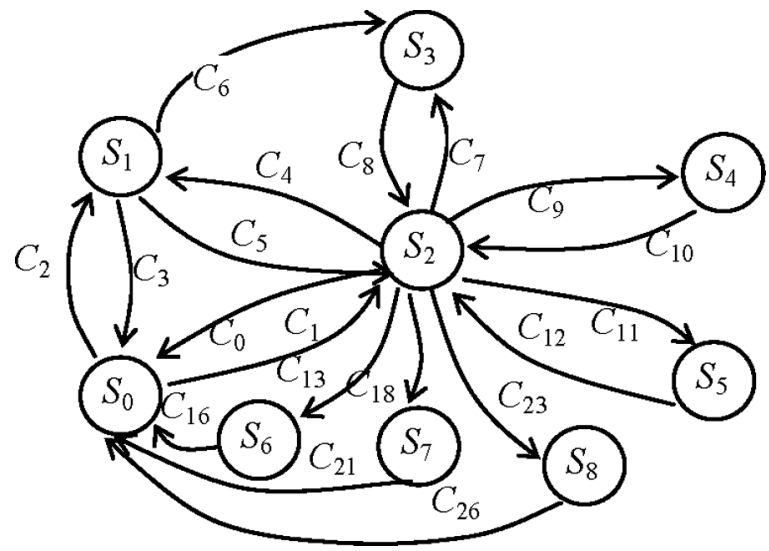

Figure 2 The diagram $\mathrm{b}$ of system running convert state

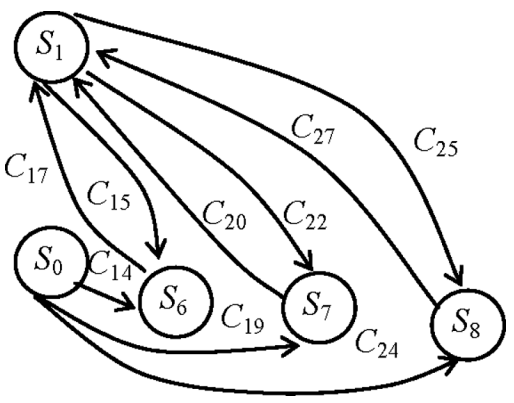

\section{Conclusions}

The energy consumption of WSN nodes is related to many factors, in this paper, we conducted in-depth research of the method of transferring state based on the time of events occurrence, but it is only from the point of view of its working state transition. Because of the uncertain factors of the event time, which is related to the amount of information to be delivered and the distance between nodes, besides, these parameters are dynamical, a prediction must be needed. So, in this paper, the amount of information to be delivered is predicted by PSO and is amended by Kalman filtering. Besides, a grey multistage model with a buffer operator is used to get the distance between nodes. Approximate reasoning based on vague set is applied to reasoning the time values of sensor networks' uncertain events. The implement of these algorithms increased the burden on the CPU operation, but, comparing the energy consumption of the RF communication to the microprocessor, there is a difference of two orders of magnitude, so that the later is less than $5 \%$ of the former. On that account, the aim to save nodes' energy consumption effectively is achieved successfully.

\section{Acknowledgements}

This work is supported by National Nature Science Foundation under Grant 61170060, Anhui Province key projects funded colleges and universities talent, Anhui Province academic and technical leader of academic research activities funded project and Anhui Province academic and technical leader backup candidate academic and research activities funded (2015D046), Top Talents funded projects in Anhui Universities (gxbjZD2016044) and National Nature Science Foundation under Grant (61170060).

\section{References}

Bakr, B.A. and Lilien, L. (2011) 'A quantitative comparison of energy consumption and WSN lifetime for LEACH and LEACH-SM', Distributed Comicroprocessorting Systems Workshops (ICDSW), Minneapolis, pp.182-191.

Bein, D., Wen, Y., Phoha, S., Madan, B.B. and Ray, A. (2011) 'Distributed network control for mobile multi-modal wireless sensor networks', Journal of Parallel and Distributed Comicroprocessorting, Vol. 45, No. 3, pp.460-470. 
Bhatti, K., Belleudy, C. and Auguin, M. (2010) 'Power management in realtime embedded systems through online and adaptive interplay of DPM and DVFS policies', Proc. of IEEE/IFIP International Conference on Embedded and Ubiquitous Comicroprocessorting, IEEE Computer Society, Washington DC, pp.184-191.

Ciszkowski, T., Dunajewski, I. and Kotulski, Z. (2011) 'Reputation as optimality measure in wireless sensor network-based monitoring systems', Probabilistic Engineering Mechanics, Vol. 26, No. 1, pp.67-75.

Ehsan, S. and Hamdaoui, B. (2012) 'A survey on energy-efficient routing techniques with QoS assurances for wireless multimedia sensor networks', IEEE Communications Surveys \& Tutorials, Vol. 14, No. 2, pp.265-278.

Eu, Z.A., Tan, H-P. and Seah, W.K.G. (2011) 'Design and performance analysis of MAC schemes for wireless sensor networks powered by ambient energy harvesting', Ad Hoc Networks, Vol. 9, pp.300-323.

Girban, G. and Popa, M. (2010) 'A glance on WSN lifetime and relevant factor for energy consumption', 2010 International Joint Conference on Comicroprocessortational Cybernetics and Technical Informatics (ICCC-CONTI), IEEE, pp.523-528.

Incel, O.D, van Hoesel, L., Jansen, P. and Having, P. (2011) 'MC-LMAC: A multi-channel MAC protocol for wireless sensor networks', Ad Hoc Networks, Vol. 9, pp.73-94.

Kamyabpour, N. and Hoang, D.B. (2010) 'A hierarchy energy driven architecture for wireless sensor networks', 24th IEEE International Conference on Advanced Information Networking and Applications (AINA), Australia, pp.668-673.
Kamyabpour, N. and Hoang, D.B. (2012) 'Statistical analysis to extract effective parameters on overall energy consumption of wireless sensor network (WSN)', Proceedings of the 2012 13th International Conference on Parallel and Distributed Comicroprocessorting, Applications and Technologies, IEEE Comicroprocessorter Society, Beijing, pp.20-23.

Molina, G. and Alba, E. (2011) 'Location discovery in wireless sensor networks using metaheuristics', Applied Soft Comicroprocessorting, Vol. 11, No. 1, pp.1223-1240.

Raghuram, P. and Venkatesh, V. (2012) 'Enhancing mine safety with wireless sensor networks using zigbee technology', Journal of Theoretical and Applied Information Technology, Vol. 37, No. 2, pp.261-267.

Rizvandi, N.B., Taheri, J. and Zomaya, A.Y. (2011) 'Some observations on optimal frequency selection in DVFS-based energy consumption minimization', J. Parallel Distrib. Comicroprocessort., Vol. 71, pp.1154-1164.

Sun, F. and Shayman, M. (2010) 'Prolong network lifetime via partially controlled node deployment and adaptive data propagation in WSN', Proc. IEEE Intl. Conf. on Information Sciences and Systems (CISS), Vol. 1, March, pp.226-231.

Tan, W.L., Lau, W.C. and Yue, O. (2012) 'Performance analysis of an adaptive, energy-efficient MAC protocol for wireless sensor networks', Journal of Parallel and Distributed Comicroprocessorting, Vol. 72, pp.504-514. 